\title{
UNA RECONSTRUCCIÓN CONJUNTISTA DE LA SEMÁNTICA DE FREGE *
}

JOSÉ LUIS ROLLERI

UNIVERSTAD MichOACANA

MORELIA

\section{Introducción}

En este escrito ofrecemos una reconstrucción conjuntista de la teoría semántica de Frege. Utilizamos el metasistema de Will Harris ${ }^{1}$ para hacer una axiomatización conjuntista informal o axiomatización informal definiendo un predicado conjuntista de la semántica fregeana.

El usar la teoría de conjuntos en la reconstrucción nos permite exhibir la estructura matemática de la teoría de Frege. La adecuación material de nuestra reconstrucción se muestra al determinar que los axiomas expresan las tesis que conforman la semántica fregeana. No modificamos en ningún respecto importante la semántica de Frege; creemos que nuestra versión de ella, si no está del todo de acuerdo con la letra de los trabajos de Frege, sí lo está con su espíritu.

Procedemos de la siguiente manera: primero explicamos brevemente qué se entiende por una axiomatización informal definiendo un predicado conjuntista; después definimos de esa manera -o sea, conjuntistamente- el predicado " $X$ es una semántica fregeana"; por último, pretendemos mostrar que nuestra reconstrucción axiomática se adecúa materialmente a la teoría semántica de Frege.

\section{La axiomatización conjuntista}

Cuando aquí decimos "reconstrucción axiomática" no queremos referirnos al tipo de reconstrucción como la que hizo Hilbert de la geometría euclideana. No estamos hablando, pues, de la axiomatización clásica a la Hilbert; más bien estamos refiriéndonos a un tipo de construcción conjuntista que algunas veces han llamado "axiomatización". Patrick

- Este escrito es parte de un trabajo más amplio que se publicará simultáneamente en Teorema.

1 “A Formal Metasystem for Frege's Semantics", Notre Dame Journal of Formal Logic, vol. XVI, núm. 1, 1975. 
Suppes, por ejemplo, dice: "El meollo del procedimiento para axiomatizar teorías dentro de la teoría de conjuntos puede ser descrito muy brevemente: axiomatizar una teoría es definir un predicado en función de nociones de la teoría de conjuntos. Un predicado así definido se llama un predicado conjuntista [set-theoretic]." a

Axiomatizar una teoría en este sentido significa, pues, definir en términos de la teoría de conjuntos un predicado apropiado. Así, por ejemplo, para axiomatizar la teoría de grupos se introduce una definición del predicado conjuntista "es un grupo". A las estipulaciones que componen la definición se les llama "axiomas" de la teoría. Un axioma es, así, sólo una parte de la definición de un predicado conjuntista. A la suma de las relaciones, si son funciones, entre los elementos de un conjunto introducido que describen los axiomas se le llama la "estructura matemática" de la teoría. Por un modelo se entiende aquí simplemente una entidad que satisface el predicado conjuntista; es decir, que cumple con los axiomas.

Si el prédicado conjuntista es introducido dentro de una teoría axiomática de conjuntos, se habla de una axiomatización formal; en caso contrario, se habla de una axiomatización informal. Nuestra axiomatización de la teoría de Frege será informal, pues no haremos uso de ninguna teoria axiomática de conjuntos.

Como ilustración damos una axiomatización conjuntista de la teoría de grupos:

$\mathrm{X}$ es un grupo si y sólo si existen $\mathrm{B}$ y $o$ tal que:

(1) $\mathrm{X}=\langle\mathrm{B}, o\rangle$;

(2) $B$ es un conjunto no vacío;

(3) $o$ es una función con dominio $=\mathrm{B} \times \mathrm{B}$ y codominio $\subseteq \mathrm{B}$;

(4) para toda $a, b$ y $c$ en $\mathrm{B}: a \circ(b \circ c)=(a \circ b) \circ c$;

(5) para toda $a$ y $b$ en $\mathrm{B}$ existe $c$ en $\mathrm{B}$ tal que $a=b o c$;

(6) para toda $a$ y $b$ en $\mathrm{B}$ existe $c$ en $\mathrm{B}$ tal que $a=c o b$.

Cualquier entidad que cumpla con las seis estipulaciones de la definición anterior es un grupo o, en otras palabras, es un modelo de la teoría de grupos así axiomatizada. De esta manera podemos decir significativamente que todos y sólo los grupos son modelos de la teoría axiomática de grupos. Esta afirmación parece circular, pero como explica Stegmüller: "Si ... una axiomatización de la teoría de grupos es entendida como la introducción del predicado conjuntista ' $\mathrm{X}$ es un grupo',

2 Introduction to Logic, D. Van Nostrand, N. J., 1975, S 12.2. Véase también, P. Suppes, Set-theoretic Structures in Science, vol. I (versión mimeográfica); Institute for Mathematical Studies in the Social Sciences, Stanford University, 1967. 
y cualquier cosa que satisface este predicado es llamado un grupo, entonces el enunciado asombrosamente simple 'todos los grupos y sólo los grupos son modelos de la axiomatización de la teoría de grupos', no es alguna clase de pretensión circular, sino más bien una especificación absolutamente precisa de los modelos de la teoria de grupos axiomatizada ..." 3

De la misma manera decimos que cualquier cosa que satisfaga el predicado conjuntista " $Y$ es una semántica fregeana simplificada" definido en el parágrafo 4, es una semántica fregeana simplificada o un modelo de cierta simplificación de la teoría semántica de Frege.*

\section{Axiomatización de la semántica de Frege}

Nuestra versión de la teoría semántica de Frege está conformada por dos supuestos metafísicos que subyacen a tal teoría. Como Harris ha señalado, ${ }^{4}$ un supuesto básico de la semántica fregeana consiste en que el lenguaje tiene una estructura idéntica, o al menos muy semejante a la estructura del mundo que describe. Hay, para Frege, un correlato ontológico, objetivo del lenguaje; tal contrapartida ontológica del lenguaje es el "reino del Bedeutung". ${ }^{5}$ La estructura del lenguaje guarda, pues, una cercana semejanza con la estructura del reino del Bedeutung. Este supuesto lleva a Frege, anota Harris, a postular un tipo de entidad para cada tipo básico de expresión. Así, el tipo de Bedeutung que corresponde a las expresiones completas son objetos, mientras que el correspondiente a las expresiones incompletas son funciones. Según el tipo de expresión es, pues, el tipo de Bedeutung.

3 The Structure and Dynamics of Theories, Springer Verlag, Nueva York, 1976, p. 35.

4 Op. cit., p. 89.

5 Frege distinguió en varios lugares entre el Sinn y el Bedeutung de una expresión. "Sinn" se traduce literalmente por "sentido", pero la traducción de "Bedeutung" presenta dificultades. Su traducción literal es "significado"; sin embargo, pocas veces se ha traducido así. H. Padilla lo traduce por "significado" en Conceptografía. Los fundamentos de la aritmética, otros ensayos filosoficos, (UNAM, 1972); en cambio, U. Moulines lo traduce por "referencia" en Estudios sobre semántica (Ariel, Barcelona, 1971); y E. Rabossi y E. Bulygin lo traducen por "denotación" en T. M. Simpson (comp.), Semántica filosófica: problemas y discusiones (Siglo XXI, Bs. As., 1973). En las traducciones inglesas de los trabajos de Frege encontramos una situación similar. P. Geach y M. Black traducen "Bedeutung" por "reference" en Translations from the Philosophical Writings of Gottlob Frege (Oxford, 1960), mientras que A. Church usa "denotation" en Introduction to Mathematical Logic, (Princeton UP, Princeton, N. J., 1965); R. Carnap propuso traducir "Bedeutung" por "nominatum" y B. Russell usó "meaning" por "Sinn". Nosotros preferimos conservar el término original alemán de Frege.

- En el $\S 4$, que no aparece aquí, mostramos que una formulación estándar de la lógica de primer orden es un modelo de una modificación de la teoráa semántica de Frege que prescinde del sentido. 
De manera similar, encontramos en Frege un segundo reino de entidades relacionadas con el lenguaje, a saber, el reino del sentido (Sinn). Para Frege una expresión expresa directamente un sentido y éste, a su vez, está conectado con un Bedeutung; la relación que existe entre una expresión y su Bedeutung no es, pues, directa, ya que es a través del sentido que una expresión bedeute ${ }^{6}$ una entidad. ${ }^{7}$ Con esta tesis semántica Frege postula el reino del sentido, reino intermediario entre el lenguaje y el reino del Bedeutung.

En la reconstrucción de la teoría de Frege postulamos dos entidades correspondientes a los dos reinos anotados. Con tales entidades construimos dos estructuras, a las que agregamos una tercera para el lenguaje. Relacionamos las estructuras entre sí por medio de funciones; tenemos así tres funciones: una para la relación directa entre la estructura del lenguaje y la del sentido; otra para la conexión entre la estructura del sentido y la del Bedeutung; una tercera para la relación indirecta entre la estructura del lenguaje y la del Bedeutung.

Nuestra axiomatización intuitiva por definición de un predicado conjuntista de la teoria semántica de Frege es la siguiente:

$X$ es una semántica fregeana si y sólo si existen $\mathbf{L}, \mathbf{S}, \mathbf{B}, \tau, \rho$ y $\sigma$ tales que

(1) $X=\langle\mathbf{L}, \mathbf{S}, \mathbf{B}, \tau, \rho, \sigma>$;

(2) $\mathrm{L}=\langle E, N, I, \alpha, \beta\rangle$ tal que

(2.1) $E$ es un conjunto no vacío,

(2.2) $N \subset E ; I \subset E ; N \cup I=E$ y $N \cap I$ es vacia;

(2.3) $\alpha$ es una función tal que el dominio $(\alpha) \subset N \times I$ y el codominio $(\alpha) \subset N$;

(2.4) $\beta$ es una función tal que el dominio $(\beta) \subset N \times I \times N$ y el codominio $(\beta) \subset N$;

(3) $\mathbf{S}=\left\langle S, S_{n}, S_{i}, \gamma, \psi\right\rangle$ tal que

(3.1) $S$ es un conjunto no vacío;

(3.2) $S_{n} \subset S ; S_{i} \subset S ; S_{n} \cup S_{i}=S \quad$ y $S_{n} \cap S_{i}$ es vacía;

(3.3) $\gamma$ es una función tal que el dominio $(\gamma) \subset S_{n} \times S_{i}$ y el codominio $(\gamma) \subset S_{n}$

(3.4) $\psi$ es una función tal que el dominio $(\psi) \subset S_{n} \times S_{i} \times S_{n}$ y el codominio $(\psi) \subset S_{n}$;

(4) $\mathbf{B}=\langle B, O, F, \omega, \lambda\rangle$ tal que

(4.1) $B$ es un conjunto no vacio;

(4.2) $O \subset B ; F \subset B ; O \cup F=B$ y $O \cap F$ es vacía;

6 "Bedeute" es el verbo asociado a "Bedeutung"; su traducción literal es "significa".

7 Véase Gottlob Frege, "Sobre sentido y referencia", traducción de Vbber Sinn und Bedeutung por Ulises Moulines en Estudios sobre semántica, Ariel, Barcelona, 1971. 
(4.3) $\omega$ es una función tal que el dominio $(\omega) \subset O \times F$ y el codominio (w) $\subset O$;

(4.4) $\lambda$ es una función tal que el dominio $(\lambda) \subset O \times F \times O$ y el codominio $(\lambda) \subset O$;

(5) $\tau: \mathbf{L} \rightarrow \mathbf{S}$ definida asi:

para cada $e \varepsilon E, \tau(c) \varepsilon S$

para cada $n \in N, \tau(n) \varepsilon S_{n}$

para cada $i \varepsilon I, \tau(i) \varepsilon S_{i}$

para cada $<n, i>\varepsilon N \times I$,

$$
\tau(\alpha(<n, i>))=\gamma(\tau(n), \tau(i))
$$

para cada $<n_{1}, i, n_{2}>\varepsilon N \times I \times N$,

$\tau\left(\beta\left(<n_{1}, i, n_{2}>\right)\right)=\psi\left(\tau\left(n_{1}\right), \tau(i), \tau\left(n_{2}\right)\right) ;$

(6) $\rho: \mathbf{S} \rightarrow \mathbf{B}$ definida así;

para cada $s \& S, \rho(s) \varepsilon B$;

para cada $s_{n} \varepsilon S_{n}, \rho\left(s_{n}\right) \varepsilon O$;

para cada $s_{i} \varepsilon S_{i}, \rho\left(s_{i}\right) \varepsilon F$;

para cada $\left\langle s_{n}, s_{i}>\varepsilon S_{n} \times S_{i}\right.$,

$\rho\left(\gamma\left(<s_{n}, s_{i}>\right)\right)=\omega\left(\rho\left(s_{n}\right), \rho\left(s_{i}\right)\right)$;

para cada $<s_{n_{1}}, s_{i}, s_{n_{2}}>_{\varepsilon} S_{n} \times S_{i} \times S_{n}$,

$\rho\left(\psi\left(<s_{n_{1}}, s_{i}, s_{n_{2}}>\right)\right)=\lambda\left(\rho\left(s_{n_{1}}\right), \rho\left(s_{i}\right), \rho\left(s_{n_{2}}\right)\right) ;$

(7) $\sigma=\rho \circ \tau$ definida así:

para cada $e \varepsilon E, \sigma(e)=\rho(\tau(e)) \varepsilon B$;

para cada $n \varepsilon N, \sigma(n)=\rho(\tau(n)) \varepsilon O$;

para cada $i \varepsilon I, \sigma(i)=\rho(\tau(i)) \varepsilon F$;

para cada $\langle n, i>\varepsilon N \times I$,

$\sigma(\alpha(<n, i>))=\omega(\sigma(n), \sigma(i)) ;$

para cada $\left\langle n_{1}, i, n_{2}>\varepsilon N \times I \times N\right.$,

$\sigma\left(\beta\left(<n_{1}, i, n_{2}>\right)\right)=\lambda\left(\sigma\left(n_{1}\right), \sigma(i), \sigma\left(n_{2}\right)\right)$.

Debemos ahora especificar qué es lo que representa cada uno de los componentes de las estructuras (2), (3) y (4) de la definición. En la estructura $\mathbf{L}, E$ representa el conjunto de expresiones de un lenguaje dado, $N$ el subconjunto de expresiones completas o nombres propios (en el sentido de Frege), $I$ el subconjunto de expresiones insaturadas o incompletas (en el sentido fregeano). a representa una operación sintáctica, la operación de saturación de Frege, para formar expresiones completas a partir de expresiones completas e incompletas. $\beta$ representa también una operación sintáctica, igualmente de saturación, para construir expresiones completas.

En $S, S$ representa el reino del sentido, $S_{n}$ el subconjunto de los sentidos correspondientes a los nombres propios y $S_{\mathbf{i}}$ el de los sentidos que corresponden a las expresiones incompletas. $\gamma$ y $\psi$ representan operacio- 
nes para los sentidos, por medio de las cuales se forman sentidos complejos en base a unos simples.

En la estructura $B$ tenemos que $B$ representa el reino del Bedeutung, $O$ el subconjunto de los objetos (en el sentido de Frege) y $F$ el subconjunto de las funciones (en el sentido fregeano). $\omega$ y $\lambda$ representan operaciones entre entidades: las que hay, para Frege, entri? los objetos y las funciones cuando aquéllos "caen bajo" (istas

Las funciones de los axiomas (5), (6) y (7) son morfismos u liomomorfismos, es decir, "trasladan" las operaciones de una estructura a otra. Estos morfismos exhiben las conexiones entre las estructuras. La función $\sigma$ es la composición de las funciones $\rho$ y $\tau$.

Las relaciones establecidas por los axiomas entre los conjuntos, y los miembros de éstos, de cada una de las estructuras, así como entre las estructuras mismas, especificadas por los morfismos, representan la estructura matemática de la teoría.

\section{Adecuación material de la axiomatización}

Dada la definición del predicado conjuntista " $X$ es una semántica fregeana", podemos ver si tal definición se adecúa materialmente a la teoría semántica de Frege.

Para empezar notemos las distinciones a nivel lingüístico, al nivel de los sentidos y al nivel de las entidades que establecen las cláusulas (2.2), (3.2) y (4.2), respectivamente. (2.2) dice que todo nombre propio es una expresión, que toda expresión insaturada es una expresión, que ninguna expresión es un nombre propio y una expresión incompleta y que toda expresión o es un nombre propio o es una expresión incompleta. Así tenemos la distinción exhaustiva de las expresiones en dos categorías excluyentes. Por su parte, (3.2) establece una distinción entre el sentido de los nombres propios y el de las expresiones insaturadas, distinción paralela a la existente, en la teoría de Frege, entre las expresiones completas e incompletas, y entre los objetos y las funciones. Frege no dio importancia a una tal distinción entre los sentidos; sin embargo, sugirió que a la instauración de las expresiones incompletas debía corresponder una instauración análoga en los sentidos. Por último, (4.2) expresa que hay dos tipos de Bedeutung, a saber, objetos y funciones, que nada es objeto y función y que cualquier entidad o es objeto o es función. De esta manera, (4.2) establece la fundamental distinción ontológica de Frege entre los objetos y las funciones. ${ }^{8}$

La función $\tau$ del axioma (5) representa la relación entre las expresio

8 Véase "Sobre concepto y objeto" y "¿Qué es una función?" 
nes y sus sentidos. (5) establece, entre otras cosas, que toda expresión tiene un sentido y sólo un sentido, ya que $\tau$ es una función. Esto concuerda con Frege, quien afirma que: "La conexión regular entre el signo, su sentido y su referencia es tal, que al signo le corresponde un determinado sentido y a éste, a su vez, una determinada referencia, mientras que a una referencia (a un objeto) no le corresponde solamente un signo." $9 \mathrm{El}$ que la función $\tau$ no sea inyectiva atiende a la posibilidad que acepta Frege de que dos expresiones tengan el mismo sentido: "El mismo sentido puede expresarse en diferentes lenguas, e incluso en la misma, de diversas maneras." ${ }^{10}$ Decidir si la función $\tau$ es suprayectiva (esto es, que la imagen coincide con el codominio) o no, es difícil, puesto que apunta a la controvertida cuestión del platonismo en Frege. ${ }^{11}$ Decir que $\tau$ no es suprayectiva equivale a afirmar que hay sentidos no conectados con ninguna expresión, a admitir un mundo platónico independiente del lenguaje. Decir que $\tau$ sí es suprayectiva equivale a afirmar que todo sentido está relacionado con alguna expresión. Nosotros dejamos esta cuestión abierta, pues rebasa nuestros propósitos en este escrito.

El hecho de que la función $\tau$ sea un morfismo significa una importante tesis semántica de Frege, a saber, que el sentido de una expresión compuesta es una función del sentido de sus componentes. Precisamente es esto lo que afirman las igualdades del axioma (5).

Por su parte, la función $\rho$ del axioma (6) representa la relación entre sentido y Bedeutung. Que $\rho$ sea una función significa que a un sentido no le corresponden dos entidades como Bedeutungen, de manera tal que el sentido determina unívocamente el Bedeutung; también significa que a cada sentido le corresponde un Bedeutung, lo cual no concuerda con la tesis semántica de Frege según la cual hay expresiones que carecen de Bedeutung, lo que sólo puede deberse, en la teoría fregeana, a que no existe la relación entre sentido y Bedeutung. Nosotros aqui "idealizamos" la teoría de Frege eliminando esta tesis, para hacer corresponder a cada sentido un Bedeutung, apoyados en la afirmación de Frege de que en un lenguaje "lógicamente perfecto" no debería haber expresiones sin Bedeutung.

La función $\rho$ no es inyectiva, puesto que en la semántica fregeana un sólo y el mismo Bedeutung puede corresponder a dos expresiones distintas, como es el caso en el famoso ejemplo de Frege de las expresiones "la estrella matutina" y "la estrella vespertina". Así, dado que en la semántica de Frege es vía sentido como una expresión designa su Bedeu-

9 "Sobre sentido y referencia", op. cit., p. 52.

10 Loc. cit.

11 Véase, por ejemplo, E. D. Klemke (ed.), Essays on Frege. University of Illinois Press, Urbana, 1968, 1a. parte. 
tung, permitimos que la función $\rho$ relacione una misma entidad con dos sentidos diferentes. La función $\rho$ tampoco es suprayectiva; esto significa que hay entidades que no están conectadas con alguna expresión, es decir, entidades no nombradas. El axioma (6) expresa además que el sentido de un nombre propio bedeute un objeto y el de una expresión insaturada bedeute una función. De esta manera se hace la distinción entre el Bedeutung correspondiente a las expresiones completas y el que corresponde a las expresiones incompletas. Que la función $\rho$ sea un morfismo significa que el Bedeutung de un sentido compuesto es una función del Bedeutung de sus componentes.

En el axioma (7) tenemos que la función $\sigma$ representa la relación indirecta entre una expresión y su Bedeutung. Esta última función, composición de $\tau$ y $\rho$, expresa que a cada expresión corresponde un y sólo un Bedeutung. Por supuesto, la composición $\sigma$ no es suprayectiva ni inyectiva. Que $\sigma$ no sea inyectiva significa que una misma entidad puede corresponder a dos expresiones distintas.

El hecho de que $\sigma$ sea una composición de $\tau$ y $\rho$ significa la importante tesis fregeana de que es a través del sentido como una expresión designa su Bedeutung. Además, la fundamental tesis semántica de Frege de que el Bedeutung de una expresión compuesta es una función de los Bedeutung de sus componentes está expresada por las igualdades del axioma (7), a saber, $\sigma(\alpha(<n, i>))=\omega(\sigma(n), \sigma(i))$ y $\sigma\left(\beta\left(<n_{1}, i\right.\right.$, $\left.\left.n_{2}>\right)\right)=\lambda\left(\sigma\left(n_{1}\right), \sigma(i), \sigma\left(n_{2}\right)\right)$. Por ejemplo, la primera dice que el Bedeutung de la expresión compuesta $a(<n, i\rangle)$ es una función de los $B e$ deutungen de las expresiones $n$ e $i$. Tenemos además que, como Harris lo ha mostrado, ${ }^{12}$ el morfismo $\sigma$ nos da el principio de sustitubilidad de los idénticos, el cual afirma que, dado que dos expresiones tienen el mismo Bedeutung, se puede sustituir una por la otra en una expresión compuesta sin cambiar el Bedeutung de ésta. Así, supóngase que:

$$
\sigma\left(n_{1}\right)=\sigma\left(n_{2}\right)
$$

entonces

$$
\omega\left(\sigma\left(n_{1}\right), \sigma(i)\right)=\omega\left(\sigma\left(n_{2}\right), \sigma(i)\right),
$$

de donde, por el axioma (7)

$$
\sigma\left(\alpha\left(<n_{1}, i>\right)\right)=\sigma\left(\alpha\left(<n_{2}, i>\right)\right) .
$$


Esta derivación muestra que el principio de sustitubilidad de los idénticos vale en general.

Recapitulemos las tesis semánticas de Frege hasta aquí anotadas. Tenemos:

(i) cada expresión tiene un sentido;

(ii) cada expresión tiene un Bedeutung;

(iii) el sentido determina el Bedeutung;

(iv) el Bedeutung de una expresión completa (o nombre propio) es un objeto, mientras que el de una expresión incompleta (o insaturada) es una función;

(v) el sentido de una expresión compuesta es una función del sentido de sus componentes;

(vi) es a través del sentido como una expresión designa su Bedeutung;

(vii) el Bedeutung de una expresión compuesta es una función del Bedeutung de sus componentes;

(viii) si dos expresiones tienen el mismo Bedeutung, podemos, en una expresión compuesta, sustituir una por la otra sin alterar el $B e$ deutung de la expresión compuesta.

Por supuesto, las anteriores no son todas las tesis semánticas de Frege. La tesis fregeana de la distinción entre el sentido y el Bedeutung de una expresión no está expresada por ninguno de los axiomas, más bien se encuentra implícita en la definición; es más, esta distinción, y los reinos correspondientes, conforman nuestra definición conjuntista. Por otro lado, algunas tesis fregeanas no pueden formar parte de nuestra definición, debido a que, o son ontológicos, o refieren a un lenguaje particular dado. Por ejemplo, Frege sostiene que el Bedeutung de un enunciado es su valor de verdad. Pero no es posible que establezcamos esta tesis, puesto que la definición de enunciado es relativa a un lenguaje particular y nosotros no tenemos, ni podemos tener, en la reconstrucción ningún lenguaje determinado. Como puede verse, en un modelo de esta axiomatización los enunciados pertenecerían a las imágenes de $\alpha$ y $\beta$. En tal modelo podría especificarse, una vez definidos los enunciados como expresiones completas, que el Bedeutung de los enunciados es su valor de verdad, de donde sería necesario que los valores veritativos fueran elementos del conjunto $O$, lo que implicaría la tesis ontológica de Frege de que los valores veritativos son objetos. Pero lo anterior sólo puede hacerse en un modelo donde se cuente con un lenguaje determinado y con una definición de verdad para los enunciados del lenguaje, y no es una versión abstracta de la teoría semántica fregeana.

Además, en los axiomas de nuestra definición no están expresadas 
aquellas tesis de Frege que se refieren al discurso indirecto, a contextos de creencia, a contextos modales; en general, a contextos oblicuos. En estos contextos no valen ciertas tesis semánticas fregeanas, a saber, (vii) y (viii) de nuestra anterior lista. Nuestra reconstrucción está diseñada para aquellos lenguajes en los que sí valen Ias tesis (vii) y (viii); aquellos lenguajes que se han llamado 'extensionales'. 